\title{
Quantifying the health benefits of genetic tests: A clinical perspective
}

Understanding the impact of genetic tests on health outcomes and health care delivery is essential to successful translation of this new technology into clinical practice through evidence-based guidelines. Khoury et al. ${ }^{1}$ describe the importance of developing a standard framework to quantify the health benefits of genetic tests "from the population perspective." They have developed a model that considers the epidemiologic parameters of risk to demonstrate the value of adding genetic tests to general prevention approaches. This is particularly relevant, as gene discoveries regarding common chronic diseases of substantial public health burden will soon result in the development of genetic tests that assess multiple loci that predict susceptibility to disease and response to treatment. They state that the potential benefit of a genetic test can depend on the "relative probabilities of uptake [compliance] and effectiveness of interventions based on knowledge of genotype." In their model, they "assume a genotype-based intervention will be available for genotype-positive people, and the general intervention will still be available to everyone, leading to a decline in the risk of disease." The examples of genotype-specific interventions they describe include low phenylalanine diet to prevent mental retardation for individuals with PKU, and lipid-lowering therapy to prevent cardiovascular events in people with familial hypercholesterolemia.

However, genotype-specific interventions may not be necessary to derive incremental benefit from genotypic information regarding disease susceptibility or responsiveness to treatment, especially for the common chronic diseases which are due most often to the interaction of genetic and non-genetic factors (e.g., lifestyle choices, exposures, and diet). An individual with knowledge of a disease susceptibility genotype may derive greater benefit from general interventions than other individuals in the population for several reasons. (1) The general intervention may be more effective in people with a particular genotype. For example, lipid-lowering to prevent cardiovascular disease is very effective for people with familial hypercholesterolemia, since high cholesterol is their major risk factor for cardiovascular disease. (2) Individuals with susceptibility genotypes may be more compliant with the general preventive intervention. Improved compliance should improve disease management and prevention if the intervention is targeting a common disease pathway or risk factor, which is likely with the complex common diseases such as heart disease, diabetes or cancer. However, improved compliance is only possible if an individual is made aware of, understands, and appropriately perceives the risk associated with their genotype and the impact of their genotype on the severity of the disease. This concept is consistent with the widely accepted health belief model which theorizes that a person's behavior is the result of several factors that can facilitate compliance with preventive behaviors or treatment interventions, including perceived susceptibility, perceived severity, perceived efficacy and cues to action. (3) Genotype results, like other risk factors, can be incorporated into an individual's overall risk assessment for a disease (i.e., global risk assessment). The intensity of general preventive interventions can then be tailored to the overall risk. For example, global risk assessment is the current approach regarding recommendations for lipid-lowering to prevent cardiovascular disease. ${ }^{3}$ Individuals with the highest risk (having the greatest number of risk factors) have a target LDL cholesterol level of $<100 \mathrm{mg} / \mathrm{dL}$. Those with intermediate risk have a target of $<130 \mathrm{mg} / \mathrm{dL}$, and those with the lowest risk have a target of $<160 \mathrm{mg} / \mathrm{dL}$. Currently genetic information is not included in this global risk assessment strategy. However, depending on the level of risk associated with susceptibility genotypes, this information could have a significant impact on an individual's risk assessment and the preventive recommendations regarding lipid-lowering treatments.

Thus, genotype information alone has the potential to reduce disease risk even in the absence of genotype-specific interventions, and we contend that it would be valuable therefore to assess the impact of communication of genetic test results in the model proposed by Khoury et al. ${ }^{1}$ This idea is compatible with their model and simply requires modification of their concept of intervention to include test results communication. Furthermore, there may be additional reasons for considering this modification. In some cases, genotype-specific interventions may never be developed for certain genetic subtypes of common diseases. Instead, genetic discoveries that explain the biological pathways of disease may result in the development of more effective general interventions for the majority. In addition, multiplex susceptibility genetic tests are already being developed and marketed before there are genotype-specific interventions, and there is no reason to suspect this will change in the near future.

Another important area that the authors do not account for in their model is the rate of adoption of this new technology, which can impact clinical and cost-effectiveness. Rogers ${ }^{4}$ describes five characteristics of an innovation that can explain different rates of adoption, including relative advantage or the degree to which an innovation is perceived as better than the idea it supercedes: compatibility or the degree to which an innovation is perceived as being consistent with existing values, past experiences, and needs of potential adopters; complexity or the degree to which an innovation is perceived as difficult to understand and use; "trialability," or the degree to 
which an innovation may be experimented with on a limited basis; and, "observability," or the degree to which the results of an innovation are visible to others. Innovations that are perceived by individuals or organizations as having greater relative advantage, compatibility, trialability, observability and less complexity will be adopted more rapidly. Generally, genetic tests are lacking in these characteristics.

Barriers to uptake of genetic testing for disease susceptibility by consumers include fear of genetic discrimination by employers and insurers, stigmatization by friends and family, and the financial cost of genetic testing, particularly if they opt to pay out of pocket to avoid health insurers. ${ }^{5}$ Barriers to adoption of genetic tests by health professionals include lack of education and training in genetics, lack of time to incorporate genetic counseling in their practice, small numbers of genetics professionals available to provide specialty consultation, lack of proven utility of testing, and poor reimbursement for activities related to genetic testing, such as counseling and the informed consent process. ${ }^{6-11}$ In their model, the authors do not account for these factors and how they may impact adoption of genetic testing. Their model only considers the decline in disease risk after gene-based intervention, stating that risk reduction depends on "background disease risk, genotype prevalence, various risk ratios, and compliance uptakes of population and genotype-based interventions." For high rates of adoption of this technology to occur, significant systems changes are needed that would positively influence health professionals' attitudes about genetic testing and their knowledge about the genetic basis of disease, indications for testing, and interpretation of test results, as well as consumers' willingness and ability to participate in genetic testing.

The promise of genotype susceptibility information to prevent disease and improve outcomes will require clinical intervention and the rate of adoption of such testing will depend upon the societal context in which it is disseminated. We hope that Khoury and his colleagues will consider the clinical and social perspectives along with the epidemiologic perspective, as they work on theoretical and empirical analyses to assess the combination of parameters for measuring the population health benefits of genetic tests. After all, as they have stated, the completion of the human genome project is expected to usher in a new era of personalized health care and prevention based on individual genetic susceptibility to disease. Thus, an epidemiologic perspective alone will tell only part of the story.

Maren T. Scheuner, MD, MPH
RAND Corporation
Santa Monica, California

Jerome I. Rotter, MD

Cedars-Sinai Medical Center

Los Angeles, California

\section{References}

1. Khoury MJ, Jones K, Grosse SD. Quantifying the health benefits of genetic tests: The importance of a population perspective. Genet Med 2005;8:191-195.

2. Janz NK, Becker MH. The health belief model: a decade later Health Educ Q 1984, 11:1-47.

3. Expert Panel on Detection, Evaluation, and Treatment of High Blood Cholesterol in Adults. Executive summary of the third report of the national cholesterol education program (NCEP) expert panel on detection, evaluation, and treatment of high blood cholesterol in adults (adult treatment panel III). JAMA 2001;285:2486-2497.

4. Rogers EM. Diffusions of Innovation, $4^{\text {th }}$ edition. The Free Press, Simon \& Schuster, Inc., 1995.

5. Slaughter LM. Genetic information must remain private to prevent discrimination, spur research. Genet Testing 1998;2:17-20.

6. Cooksey JA, Forte G, Benkendorf J, Blitzer MG. The state of the medical geneticist workforce: Findings of the 2003 survey of American Board of Medical Genetics certified geneticists. Genet Med 2005;7:439-443.

7. Gramling R, Nash J, Siren K, Eaton C, et al. Family physician self-efficacy with screening for inherited cancer risk. Ann Fam Med 2004;2:130-132.

8. Pletcher BA, Jewett EAB, Cull WL, Brotherton SE, et al.The practice of clinical genetics: A survey of practitioners. Genet Med 2002;4:142-149.

9. Rich EC, Burke W, Heaton CJ, Haga S, et al. Reconsidering the family history in primary care. J Gen Intern Med 2004;19:273-280.

10. Suther S, Goodson P.Barriers to the provision of genetic services by primary care physicians: a systematic review of the literature. Genet Med 2003;5:70-76.

11. Yarnall KS, Pollak KI, Ostbye T, Krause KM, et al. Primary care: Is there enough time for prevention? Am J Public Health 2003;93:635-641. 